\title{
A DIY universal fraction collector
}

David Díaz $^{1}$, Ana de la Iglesia ${ }^{1}$, Francisco Barreto $^{2}$ and Ricardo Borges ${ }^{1, *}$

${ }^{1}$ Unidad de Farmacología, Facultad de Medicina and ${ }^{2}$ Servicio de Electrónica, Universidad de La Laguna, E-38200 La Laguna. Tenerife, Spain.

*Author for correspondence

email: rborges@ull.edu.es

\section{TABLE OF CONTENT}

- Catalogue of STL pieces

Table S1: Mobile platform

Table S2: Control box

Table S3: Dropper

- Cost of materials

Table S4: Hardware parts

Table S5: Electronic device

Table S6: Expansion board

- Arduino firmware

Table S7: Arduino UNO firmware files

- Mounting guidelines

Scheme S1: Expansion board

Scheme S2: Expansion board views

Scheme S3: Wiring

Scheme S4: mechanical assembly

- Mounting steps.

- Video of the full system working 


\section{Catalogue of STL pieces}

\section{Table S1: Mobile platform}

\begin{tabular}{|c|c|c|c|c|c|}
\hline Fig. & item & Image & Description & Repository file & Amount \\
\hline $2 \mathrm{~B}$ & 1 & & Prusa $^{\circledR}$ corner nut & platform-corner.stl ${ }^{(1)}$ & 4 \\
\hline $2 \mathrm{C}$ & 11 & & $\begin{array}{l}\text { Holder for the step } \\
\text { motor }\end{array}$ & $\begin{array}{l}\text { platform-motor } \\
\text { holder.stl }^{(1)}\end{array}$ & 1 \\
\hline $2 \mathrm{C}$ & 15 & & $\begin{array}{l}\text { Holder for the end- } \\
\text { limit Makerbot }{ }^{\circledR} \\
\text { switches }\end{array}$ & $\begin{array}{l}\text { platform-switch } \\
\text { holder.stl }\end{array}$ & 2 \\
\hline $2 \mathrm{C}$ & 7 & & Belt tensor & $\begin{array}{l}\text { platform-belt tensor.stl } \\
\text { (1) }\end{array}$ & 1 \\
\hline $2 \mathrm{C}$ & 8 & & Pulley for belt tensor & $\begin{array}{l}\text { platform-belt tensor } \\
\text { pulley }^{(1,2)}\end{array}$ & 2 \\
\hline $2 \mathrm{C}$ & 5 & & Carriage & platform-carriage.stl & 1 \\
\hline $2 \mathrm{C}$ & 13 & & Belt clamps & platform-belt clamp.stl ${ }^{(1)}$ & 1 \\
\hline $2 \mathrm{C}$ & 16 & & Plate drillings & DRL.tiff ${ }^{(3)}$ & 1 \\
\hline
\end{tabular}

(1) File obtained from https://www.bq.com/es/support/prusa/support-sheet.

(2) Two pieces are necessary to make the pulley and a $\varnothing 3 \mathrm{~mm}$ screw is perfect for axis.

(3) This file contains the diagram for drilling the methacrylate plate. 
Table S2: Control box

\begin{tabular}{|c|c|c|c|c|c|}
\hline Fig. & item & Image & Description & Repository file & Amount \\
\hline $3 \mathrm{~A}$ & 21 & & Box holder & controlbox-holder.stl & 2 \\
\hline $3 \mathrm{~A}$ & 22 & & Case Part A & controlbox- case A.stl & 1 \\
\hline $3 \mathrm{~A}$ & 23 & 0 & Case Part B & controlbox- case B.stl & 1 \\
\hline 3B & 24 & & Internal part & controlbox-internal part.stl & 1 \\
\hline
\end{tabular}

\section{Table S3: Dropper}

\begin{tabular}{|c|c|c|c|c|c|}
\hline Fig. & item & Image & Description & Repository file & Amount \\
\hline $4 \mathrm{~A}$ & 27 & & Dropper receptacle & $\begin{array}{l}\text { dripper-droppers } \\
\text { holder.stl }^{(1)}\end{array}$ & 1 \\
\hline $4 B$ & 28 & & Dropper holder & dripper-beam.stl & 1 \\
\hline $4 \mathrm{~A}$ & 31 & & $\begin{array}{l}\text { Stand holder for } \\
\text { dropping system }\end{array}$ & dripper-stand.stl & 1 \\
\hline
\end{tabular}

\footnotetext{
(1) This piece is conceived to accommodate six yellow pipette tips.
} 


\section{Cost of the materials}

\section{Table S4: Hardware parts}

\begin{tabular}{|c|c|c|c|c|}
\hline & Item & Description & Amount & Price $(€)$ \\
\hline $2 \mathrm{C}$ & 3 & Metal Ø-8 mm threated-rod / $200 \mathrm{~mm}$ long & 4 & 0.80 \\
\hline 2B & 4 & Metal Ø-8 $\mathrm{mm}$ rod / $500 \mathrm{~mm}$ long & 2 & 1.50 \\
\hline 2B & 2 & Metal Ø-10 mm threated-rod / $500 \mathrm{~mm}$ long & 2 & 1.30 \\
\hline $4 \mathrm{~A}$ & 30 & Metal Ø-6 mm rod $/ 250 \mathrm{~mm}$ long & 2 & 0.60 \\
\hline \multicolumn{2}{|c|}{ Not marked } & Metal Ø-3 mm nut (for pulley axis) & 1 & 0.08 \\
\hline \multicolumn{2}{|c|}{ Not marked } & Metal $\varnothing-8 \mathrm{~mm}$ nut & 20 & 1.05 \\
\hline \multicolumn{2}{|c|}{ Not marked } & Metal $\varnothing-10 \mathrm{~mm}$ nut & 16 & 1.20 \\
\hline \multicolumn{2}{|c|}{ Not marked } & Ø-3 mm screws / $20 \mathrm{~mm}$ long (for control mount) & 7 & 0.14 \\
\hline \multicolumn{2}{|c|}{ Not marked } & Ø-5 mm screws / $10 \mathrm{~mm}$ long & 4 & 0.10 \\
\hline $2 \mathrm{C}$ & 8 & $623 \mathrm{ZZ}$ bearing (inside pulley) & 1 & 0.30 \\
\hline $2 \mathrm{C}$ & 9 & GT2-6 $\mathrm{mm}$ rubber belt & $1 \mathrm{~m}$ & 1.80 \\
\hline $2 \mathrm{C}$ & 12 & GT2 aluminium gear / 20 teeth / shaft $\varnothing-5 \mathrm{~mm}$ & 1 & 0.90 \\
\hline 2B & 10 & LM8UU linear bearing & 4 & 4.40 \\
\hline \multicolumn{2}{|c|}{ Not displayed } & Clear Round Self-Adhesive Rubber Feet Tall Bumpers & 8 & 0.90 \\
\hline $2 \mathrm{C}$ & 16 & Methacrylate rectangular piece $200 \times 400 \times 10 \mathrm{~mm}$ & 1 & 0.87 \\
\hline
\end{tabular}

Table S5: Electronic device

\begin{tabular}{|cc|l|cc|}
\hline Fig. & Item & Description & Amount & Price (€) \\
\hline 2C & 6 & 17HS8401 4-lead Nema17 stepper motor & 1 & 13.00 \\
2C & 14 & Makerbot end-stop switch board & 2 & 0.80 \\
3B & 18 & $\begin{array}{l}\text { LCD Module 2004A + I2C/IIC PCF8574T board } \\
\text { adapter }\end{array}$ & 1 & 12.00 \\
3B & 19 & Rotary encoder EC11 Series with push-button switch & 1 & 1.50 \\
3D & 17 & Arduino UNO REV3 board & 1 & 12.00 \\
\hline \multicolumn{2}{r|}{ Total Cost } & $\mathbf{3 9 . 3 0}$ \\
\hline
\end{tabular}




\section{Table S6: Expansion board}

(see fractionCollector-expansionBoardSchematic.pdffile)

\begin{tabular}{|c|c|c|c|}
\hline Item & Description & Amount & Price $(€)$ \\
\hline Not marked & $\begin{array}{l}\text { Pololu A4988 Stepper Motor Driver. } \\
\text { Schematic part: U1 }\end{array}$ & 1 & 2.00 \\
\hline Not marked & $\begin{array}{l}\text { 2-way / } 2.54 \mathrm{~mm} \text { pitch PCB terminal block connector } \\
\text { Schematic parts: } \mathrm{J} 2, \mathrm{~J} 3, \mathrm{~J} 4, \mathrm{~J} 6, \mathrm{~J} 7, \mathrm{~J} 8, \mathrm{~J} 10, \mathrm{~J} 11, \mathrm{~J} 12, \mathrm{~J} 14, \mathrm{~J} 15, \mathrm{~J} 16, \mathrm{~J} 17\end{array}$ & 13 & 19.00 \\
\hline Not marked & $\begin{array}{l}\text { 36-way / } 1 \text { row / } 2.54 \mathrm{~mm} \text { pitch straight Pin Header. } \\
\text { Schematic parts: } \mathrm{J1}, \mathrm{J} 5, \mathrm{~J} 9, \mathrm{~J} 13\end{array}$ & 1 & 0.80 \\
\hline Not marked & $\begin{array}{l}4.7 \mathrm{~K} \Omega \pm 5 \% \text { / } 0.25 \mathrm{~W} / \text { through hole / axial / carbon film } \\
\text { resistor. } \\
\text { Schematic parts: R1, R2 }\end{array}$ & 2 & 0.24 \\
\hline Not marked & $\begin{array}{l}100 \mu \mathrm{F} 50 \mathrm{~V} \pm 20 \% \text { / Ø 8mm / electrolytic aluminium } \\
\text { capacitor } \\
\text { Schematic part: } \mathrm{C} 1\end{array}$ & 1 & 12.00 \\
\hline Not marked & Printed circuit board fabrication & 1 & 1.50 \\
\hline \multicolumn{3}{|c|}{ Total Cost } & 23.64 \\
\hline
\end{tabular}

\section{Arduino firmware}

\section{Table S7: Arduino UNO firmware files.}

\begin{tabular}{|l|l|}
\hline File name. & File type. \\
\hline LiquidCrystal_I2C-1.1.2 & Library \\
RotaryEncoder-1.1.0 & Library \\
encoder.h & Header \\
plate.h & Header \\
screens.h & Header \\
encoder.cpp & Source \\
plate.cpp & Source \\
screens.cpp & Source \\
fractionCollector.ino & Source \\
\hline
\end{tabular}




\section{Mounting guidelines}

Scheme S1: Expansion board
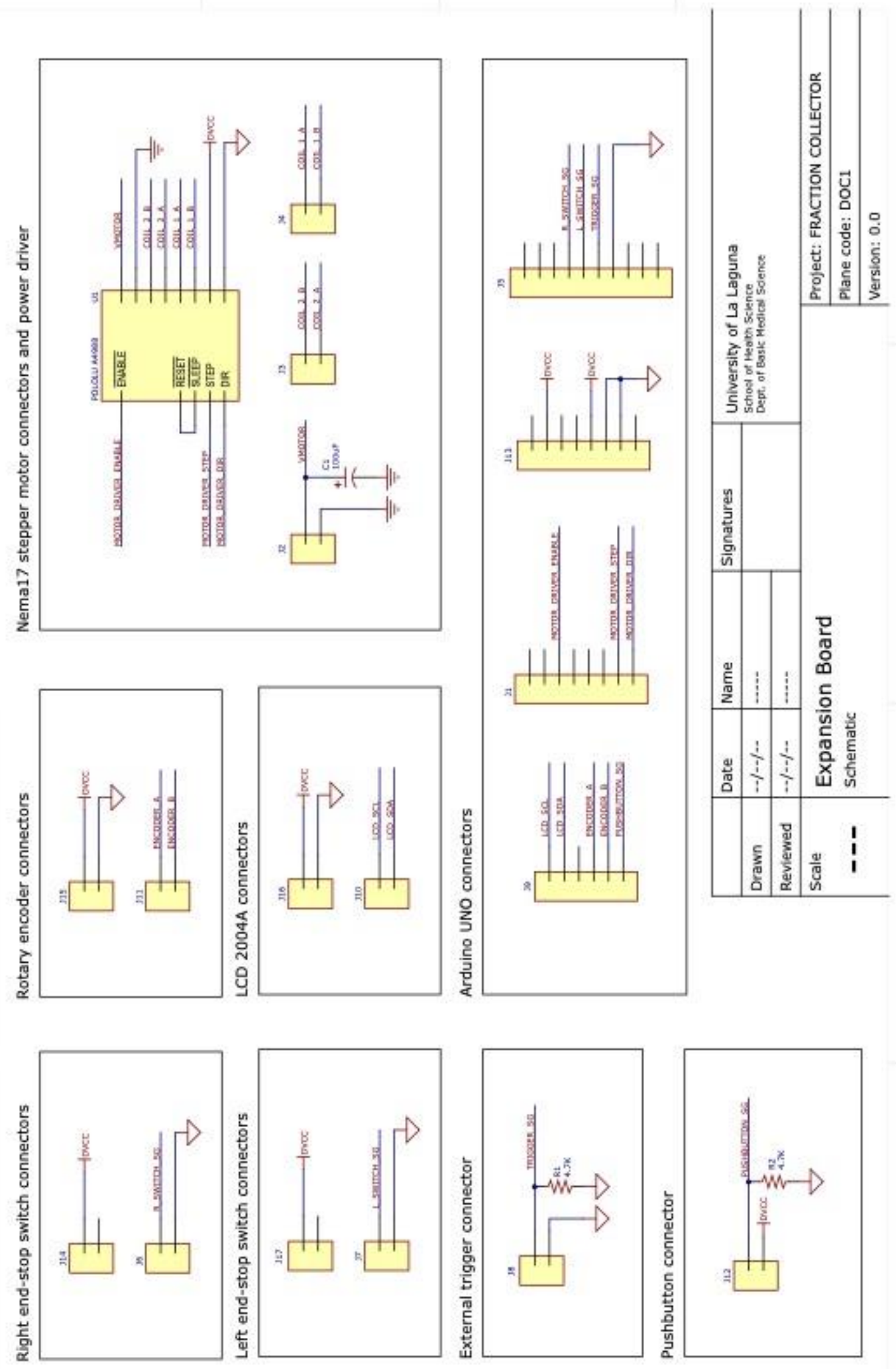
Scheme S2: Expansion board views

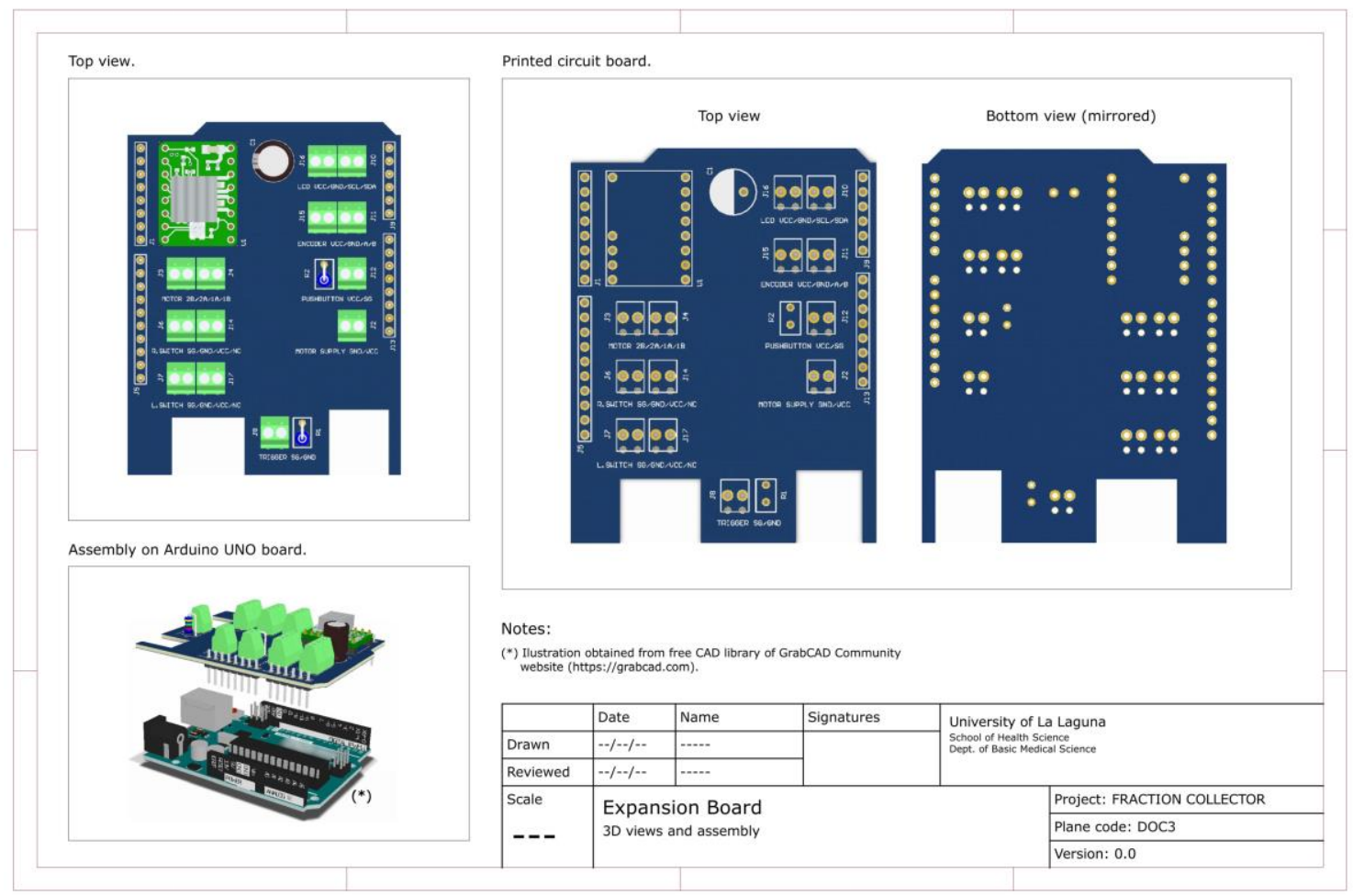

\section{Scheme S3: Wiring}

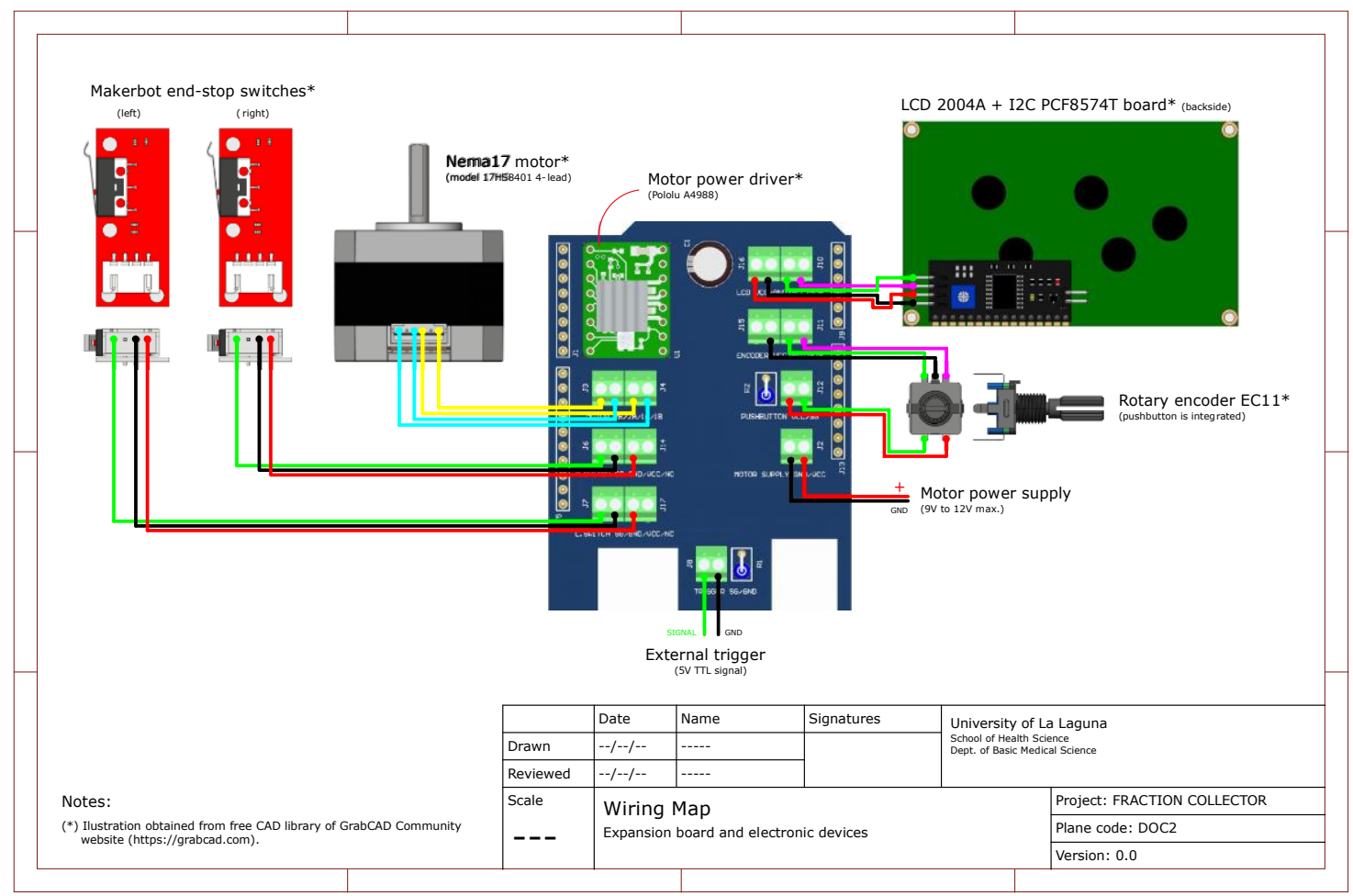


Scheme S4: mechanical assembly

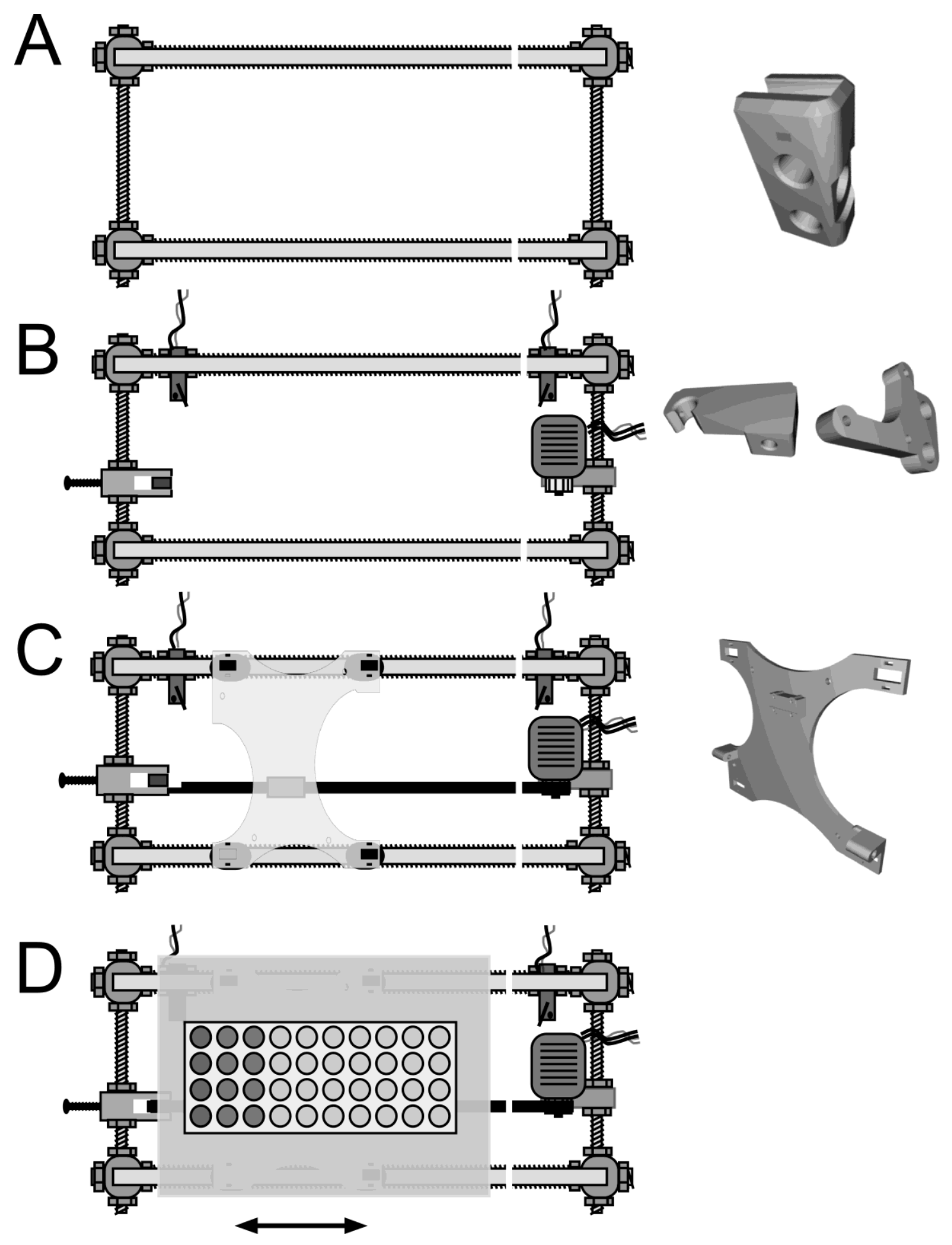

Mounting steps. A, montage of the frame. B, Adding the step motor, the stoppers and the belt tensor. C, Adding the belt and the platform with the belt clamp. D, screwing on the methacrylate platform. 
Video of the full system working 